\title{
Stratification of earth's outermost core inferred from SmKS array data
}

\author{
Satoshi Kaneshima ${ }^{1 *}$ and Takanori Matsuzawa ${ }^{2}$
}

\begin{abstract}
SmKS arrivals recorded by large-scale broadband seismometer arrays are analyzed to investigate the depth profile of $P$ wave speed $\left(V_{p}\right)$ in the outermost core. The $V_{p}$ structure of the upper $700 \mathrm{~km}$ of the outer core has been determined using SmKS waves of Fiji-Tonga events recorded at stations in Europe. According to a recent outer core model (KHOMC), the $V_{p}$ value is $0.45 \%$ slower at the core mantle boundary (CMB) than produced by the Preliminary Reference Earth Model (PREM), and the slow anomaly gradually diminishes to insignificant values at $\sim 300 \mathrm{~km}$ below the CMB. In this study, after verifying these KHOMC features, we show that the differential travel times measured for SmKS waves that are recorded by other large-scale arrays sampling laterally different regions are well matched by KHOMC. We also show that KHOMC precisely fits the observed relative slowness values between S2KS, S3KS, and S4KS (SmKS waves with $m=2,3$, and 4). Based on these observations, we conclude that SmKS predominantly reflect the outer core structure. Then we evaluate biases of secondary importance which may be caused by mantle heterogeneity. The KHOMC $V_{p}$ profile can be characterized by a significant difference in the radial $V_{p}$ gradient between the shallower $300 \mathrm{~km}$ and the deeper part of the upper $700 \mathrm{~km}$ of the core. The shallower part has a $V_{p}$ gradient of $-0.0018 s^{-1}$, which is steeper by $0.0001 s^{-1}$ when compared to the deeper core presented by PREM. The steeper $V_{p}$ gradient anomaly of the uppermost core corresponds to a radial variation in the pressure derivative of the bulk modulus, $K^{\prime}=d K / d P$. The $K^{\prime}$ value is 3.7 , which is larger by about 0.2 than that of the deeper core. The radial variation in $K^{\prime}$ is too large to have a purely thermal origin, according to recent ab initio calculations on liquid iron alloys, and thus requires a thick and compositionally stratified layering at the outermost outer core.
\end{abstract}

Keywords: Outermost core; Compositional stratification; SmKS waves; Array processing

\section{Background}

Stratification of the outermost core has been long suspected (e.g., Lay and Young 1990; Buffett and Seagle 2010) and has been recently strongly advocated by Helffrich and Kaneshima (2010) and Kaneshima and Helffrich (2013) (respectively abbreviated as HK2010 and KH2013 hereafter). The present study consolidates the propositions of HK2010 and KH2013 by investigating additional SmKS data obtained for significantly more event-array pairs and by evaluating possible mantle effects on S $m K S$ travel time and slowness observations. We analyze SmKS arrivals with integer $m$ from 2 to 5 in order to better constrain the $V_{p}$ profile of the topmost $700 \mathrm{~km}$ of the core, anticipating

\footnotetext{
*Correspondence: kane@geo.kyushu-u.ac.jp

1 Department of Earth and Planetary Science, Kyushu University, Hakozaki,

Higashi-ku, 812-8581 Fukuoka, Fukuoka, Japan

Full list of author information is available at the end of the article
}

that the results will strengthen the evidence for a compositionally stratified layer. As shown in Fig. 1, the ray paths of S2KS, S3KS, and S4KS propagate quite a long distance through the outermost part of the core. Since each SmKS possesses a distinct depth sensitivity depending both on $m$ and epicentral distance, when used in combination, $S m K S$ waves can form an ideal data set for use in determining the $V_{p}$ profile of the outermost core. In order to measure differential travel time and slowness differences between two SmKS waves, we used large-scale broadband seismometer arrays in Europe, Eastern Asia (including Japan), North and South America, Africa, and Australia.

Prior to HK2010 and KH2013, other studies also investigated the outer core structure by analyzing SmKS data. The proposed models show either slightly or rather strongly slower $V_{p}$ anomaly relative to the Preliminary Reference Earth Model (PREM) (Dziewonski and Anderson 1981) near the top of the core. For instance,

\section{是 Springer}

(c) 2015 Kaneshima and Matsuzawa. This is an Open Access article distributed under the terms of the Creative Commons Attribution License (http://creativecommons.org/licenses/by/4.0), which permits unrestricted use, distribution, and reproduction in any medium, provided the original work is properly credited. 


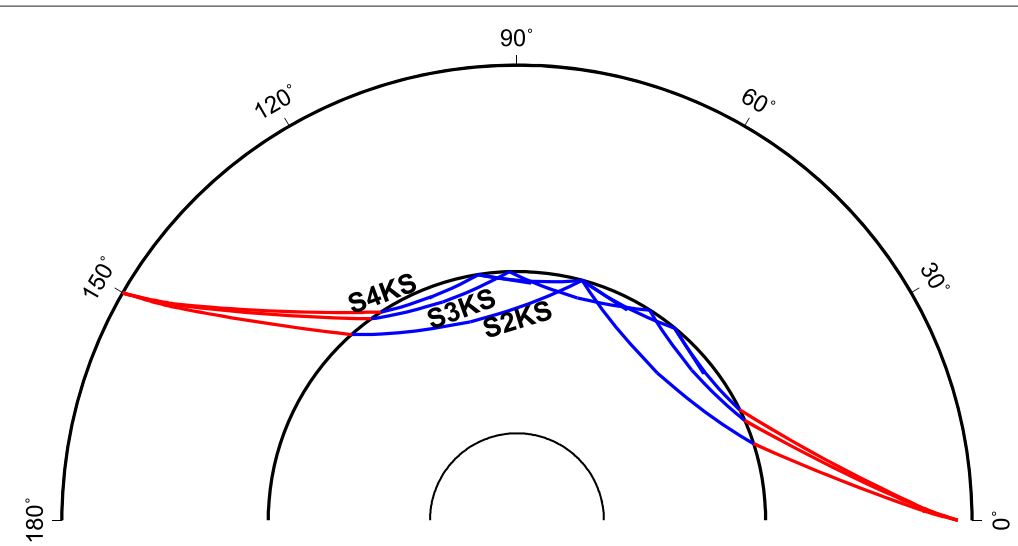

Fig. 1 SmKS ray paths. Ray paths of SmKS waves (S2KS, S3KS, and S4KS) propagating through the outermost part of the core to a receiver at a epicentral distance of $150^{\circ}$. Blue and red lines show $\mathrm{P}$ and $\mathrm{S}$ wave portions of the rays, respectively

Tanaka (2007) analyzed a composite record section of S2KS, S3KS, and S4KS, which were observed globally, and proposed a model with up to a maximum of $1.2 \%$ slower $V_{p}$ than PREM in the outermost $90 \mathrm{~km}$ of the core. His model (called Tanaka-1 hereafter) is similar to that presented in an earlier study by Garnero et al. (1993). Alexandrakis and Eaton (2010) investigated composite globally observed record sections of S2KS to S4KS for the distance range shorter than $140^{\circ}$ and showed a permissible range of $V_{p}$ profiles for the top $200 \mathrm{~km}$ of the outer core. The range of permissible models centers around slightly slower $V_{p}$ values (from $\sim 0.1$ to $0.4 \%$ ) than PREM and falls between PREM and the IASP91 velocity model (Kennett and Engdahl 1991). In this study, we also include other global $V_{p}$ models such as SP6 (Morelli and Dziewonski 2012), AK135 (Kennett et al. 1995), and another model proposed by Tanaka (2007) (called Tanaka-3 hereafter) with the models to be compared.

\section{SmKS waveform data}

S $m$ KS data are analyzed for earthquakes that occurred in several different regions and were recorded at largescale broadband stations prior to July 2014 (Table 1). The broadband seismograms in Europe are extracted from the Incorporated Research Institutions for Seismology (IRIS) and the Observatories and Research Facilities for European Seismology (ORFEUS) data management centers. Seismic stations in Japan belong to J-Array, F-net, and the tilt-meter network of Hi-net (Obara et al. 2005). Data of the seismic stations in Eastern Asia outside of Japan, the US, and Australia are extracted from the IRIS data management center (DMC). In this study, we loosely define a "large-scale array" as a group of 20 or more broadband seismometer stations with aperture lengths exceeding $\sim 1000 \mathrm{~km}$ whose seismograms can be analyzed by standard array processing methods. As we shall show later, travel times of $\mathrm{S} m \mathrm{KS}$ waves observed at individual stations may be significantly affected by the heterogeneous mantle structure, rather irrespective of the waveform quality. This makes it difficult to investigate a detailed $V_{p}$ profile of the outer core based on SmKS measurements made for individual traces. However, HK2010 and KH2013 showed that stacking seismograms over an entire large-scale array substantially suppresses the mantle effects and thereby enables us to discern the fine structure of the outermost core. In this study, we shall also show that the processing of large-scale array data enables us to precisely retrieve differential slowness between two SmKS arrivals, which helps to better constrain the outer core structure. Figure 2 shows an example of observed and computed record sections for a Fiji-Tonga event recorded at the European stations. The synthetic seismograms shown in Fig. 2b are computed for PREM using reflectivity (Kind 1979) and a method similar to that of Wang et al. (2008) (HK2010). When SmKS touches an internal caustic and is underside reflected $m-1$ times at the core mantle boundary (CMB), it suffers from a phase delay by nearly $\frac{m-1}{2} \pi$ relative to SKS (Choy 1977). The arrivals of S2KS, S3KS, S4KS, S5KS, and S6KS are clearly visible on this section and are systematically delayed with respect to the theoretical PREM predictions throughout the array.

\section{Methods and results}

\section{Array measurements}

In this study, we measure differential travel times between two different SmKS waves on stacked waveforms; those between S3KS and S2KS (called $d t^{3-2}$ hereafter), S4KS and S3KS (called $d t^{4-3}$ ), and S5KS and S3KS (called $d t^{5-3}$ ). Next, the differential time anomalies relative to the ray theoretical predictions based on PREM are computed. Since instrument corrections have negligible effects on the results, stacking and differential time measuring are performed directly on the observed broadband seismograms (HK2010). We also avoid unstable deconvolution of 
Table 1 Event list

\begin{tabular}{|c|c|c|c|c|c|c|}
\hline Event & Date & Time & Lat. N & Lon. E & $\mathrm{h}(\mathrm{km})$ & Mo (dyne cm) \\
\hline FEV1 & 2007/10/05 & 7:17:52 & -25.19 & 179.46 & 509 & $6.8 \times 10^{25}$ \\
\hline FEV2 & 2007/10/16 & $21: 5: 43$ & -25.77 & 179.53 & 509 & $1.0 \times 10^{26}$ \\
\hline FEV3 & 2008/07/03 & $3: 2: 37$ & -23.37 & -179.78 & 581 & $2.8 \times 10^{25}$ \\
\hline FEV4 & 2011/07/29 & $7: 42: 24$ & -23.79 & 179.75 & 537.0 & $1.5 \times 10^{26}$ \\
\hline FEV5 & 2007/05/06 & 21:11:53 & -19.40 & -179.35 & 676 & $6.1 \times 10^{25}$ \\
\hline FEV6 & $2012 / 01 / 24$ & 00:52: 5 & -24.977 & 178.520 & 580.3 & $4.2 \times 10^{25}$ \\
\hline FEV7 & 2008/07/19 & $22: 39: 53$ & -17.337 & -177.312 & 391. & $5.2 \times 10^{25}$ \\
\hline FEV8 & 2011/08/19 & $3: 54: 27$ & -16.522 & -177.004 & 407.9 & $2.9 \times 10^{25}$ \\
\hline FEV9 & 2008/10/19 & $5: 10: 34$ & -21.863 & -173.819 & 29.0 & $2.9 \times 10^{26}$ \\
\hline FEV10 & 2009/03/19 & $18: 17: 41$ & -23.050 & -174.660 & 34.0 & $3.4 \times 10^{27}$ \\
\hline FEV11 & 2009/11/24 & $12: 47: 16$ & -20.710 & -174.040 & 18.0 & $1.7 \times 10^{26}$ \\
\hline FEV12 & $2014 / 07 / 21$ & $14: 54: 41$ & -19.829 & -178.464 & 616. & $3.0 \times 10^{26}$ \\
\hline FEV13 & 1998/03/29 & $19: 48: 16$ & -17.552 & -179.092 & 537.5 & $6.4 \times 10^{26}$ \\
\hline KEV1 & 2013/04/26 & $6: 53: 29$ & -28.68 & -178.96 & 358.8 & $2.1 \times 10^{25}$ \\
\hline KEV2 & $2011 / 02 / 21$ & $10: 57: 52$ & -26.142 & 178.394 & 558.0 & $7.9 \times 10^{25}$ \\
\hline KEV3 & 2007/09/25 & 5:16: 1 & -30.965 & 179.998 & 417. & $2.3 \times 10^{25}$ \\
\hline KEV4 & 2009/08/18 & $21: 20: 47$ & -26.064 & -178.391 & 269.3 & $3.6 \times 10^{25}$ \\
\hline KEV5 & $2013 / 08 / 28$ & $2: 54: 41$ & -27.75 & 179.62 & 480.3 & $2.9 \times 10^{25}$ \\
\hline KEV6 & $2014 / 06 / 23$ & 19:19:16 & -30.118 & -177.67 & 20. & $2.6 \times 10^{26}$ \\
\hline AEV1 & $2000 / 04 / 23$ & $9: 27: 23$ & -28.31 & -62.99 & 609 & $3.1 \times 10^{26}$ \\
\hline AEV2 & $2005 / 03 / 21$ & $12: 23: 54$ & -24.98 & -63.47 & 579 & $2.3 \times 10^{26}$ \\
\hline AEV3 & 2008/09/03 & $11: 25: 14$ & -26.74 & -63.22 & 570 & $3.3 \times 10^{25}$ \\
\hline AEV4 & $2012 / 05 / 28$ & $5: 7: 24$ & -28.06 & -63.11 & 589.3 & $1.3 \times 10^{26}$ \\
\hline AEV5 & 2011/09/02 & 13:47:11 & -28.420 & -63.150 & 592.8 & $1.4 \times 10^{26}$ \\
\hline AEV6 & $2014 / 04 / 03$ & 1:58:30 & -20.311 & -70.576 & 24.1 & $9.6 \times 10^{25}$ \\
\hline NCEV & 2014/04/11 & 20:29:12 & 11.642 & -85.878 & 135.0 & $1.0 \times 10^{25}$ \\
\hline IEV1 & 2011/08/30 & $6: 57: 42$ & -6.36 & 126.76 & 469.0 & $2.7 \times 10^{26}$ \\
\hline IEV2 & 2010/03/20 & 14: 0:50 & -3.36 & 152.24 & 414 & $9.2 \times 10^{25}$ \\
\hline IEV3 & 2010/07/23 & 22: 8:11 & 6.72 & 123.41 & 607.0 & $1.2 \times 10^{27}$ \\
\hline IEV4 & 2013/06/13 & $16: 47: 23$ & -10.0 & 107.24 & 9.0 & $1.1 \times 10^{26}$ \\
\hline IEV5 & 2011/03/10 & 17:08:37 & -6.87 & 116.72 & 510.6 & $9.7 \times 10^{25}$ \\
\hline IEV6 & $2010 / 05 / 31$ & $19: 51: 45$ & 11.132 & 93.471 & 112. & $6.4 \times 10^{25}$ \\
\hline MEV & $2006 / 02 / 22$ & 22:19: 8 & -21.32 & 33.58 & 11 & $4.2 \times 10^{26}$ \\
\hline INEV & $2008 / 05 / 31$ & 4:37:56 & -41.290 & 80.47 & 10. & $5.1 \times 10^{25}$ \\
\hline SEV1 & $2014 / 06 / 29$ & 7:52:56 & -55.506 & -28.451 & 16.5 & $2.4 \times 10^{26}$ \\
\hline PEV1 & 2013/05/14 & $0: 32: 26$ & 18.728 & 145.287 & 602.3 & $2.1 \times 10^{26}$ \\
\hline
\end{tabular}

the source time function, which may cause some biases to the $d t^{m-n}$ (differential travel times between SmKS and $\mathrm{S} n \mathrm{KS}$ waves) and their anomalies relative to ray theoretical predictions by PREM, which are usually positive. Those biases are mostly less than $0.2 \mathrm{~s}$ for the Fiji-Tonga events used in HK2010 and KH2013 but can be larger for other events. Therefore, we evaluate biases by using synthetic seismograms (Fig. 2b). To measure these synthetic seismograms $d t^{m-n}$, we use the same array method as the data and compare them with the theoretical ray calculations based on PREM. The difference, which is usually 0.2 to $0.3 \mathrm{~s}$ but sometimes could exceed $0.5 \mathrm{~s}$ (Table 2), is regarded as the bias. For each event, $d t^{3-2}$ are measured both by picking peaks and cross-correlating the stacked waveforms after correcting for the $\frac{\pi}{2}$ phase shift due to the caustic (Choy 1977). Both S2KS and S3KS are 


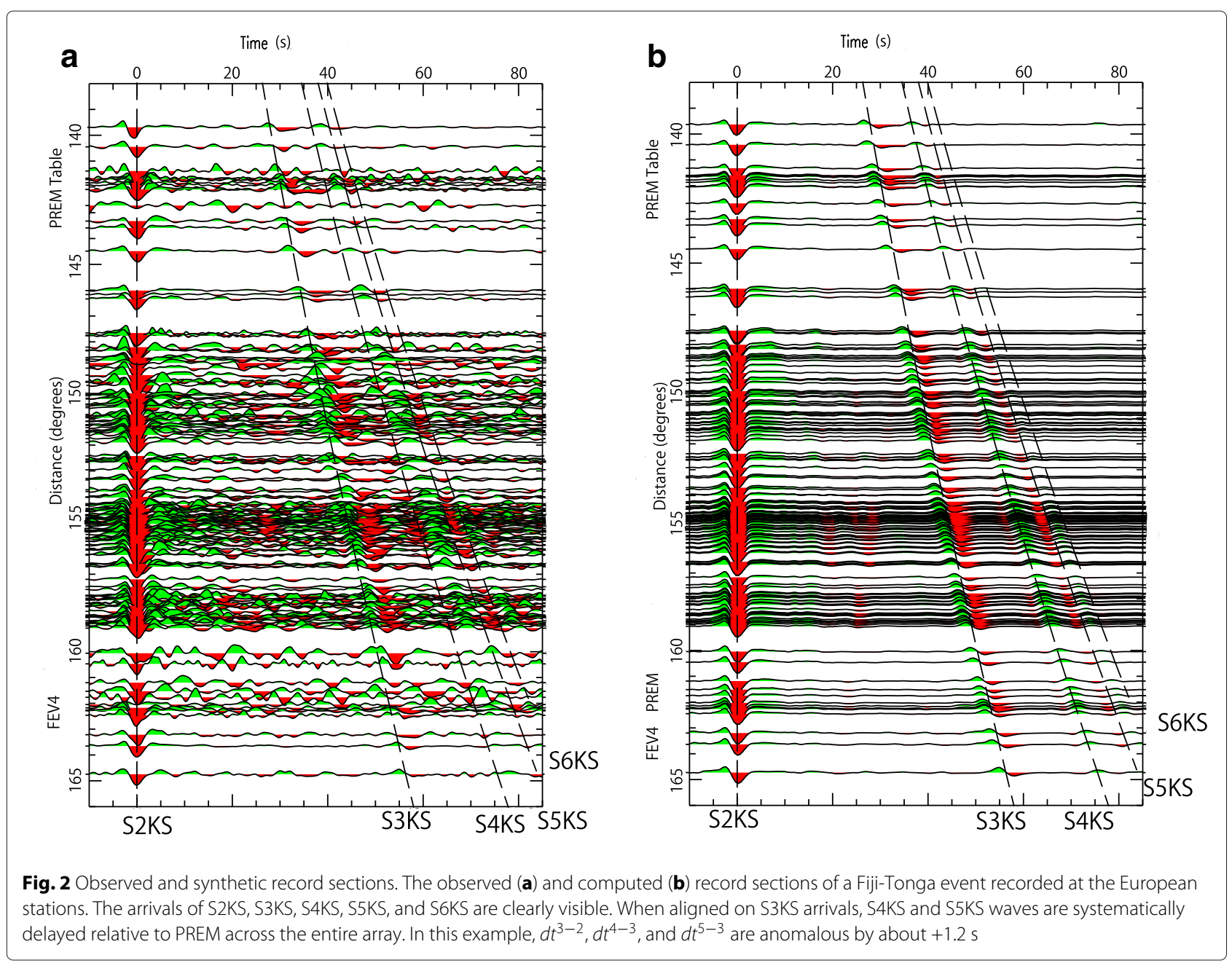

used as the alignment phase whenever the S3KS arrival is sufficiently clear, and the agreements between different alignment phases (S2KS or S3KS) and between different methods (peak-picking or cross correlating) are used for evaluating the error of each $d t^{3-2}$ measurement. The measured differential times are classified into two categories, A (high quality) and B (moderate) (Table 2). When the values obtained by different methods agree with each other, the stacked synthetic and observed seismograms usually agree quite well, and the measurements can be regarded as category $\mathrm{A}$, and have smaller errors.

In addition to the $\frac{\pi}{2}$ phase shift, waveforms of S4KS could be distorted by the interference by S5KS (Eaton and Kendall 2006) or by S3KS when epicentral distances are not large. The S5KS arrivals are not separated completely from S4KS, even when its peak, which is oppositely polarized to S3KS, can be clearly identified. Therefore, $d t^{4-3}$ and $d t^{5-3}$ are measured simply by identifying the corresponding peaks. Since alignment on S4KS arrivals is usually unstable, differential times between S4KS and S5KS (called $d t^{5-4}$ ) are calculated by subtracting $d t^{4-3}$ from $d t^{5-3}$ with error propagation. Other array measurement details are described in HK2010 and KH2013. We note that, especially for the Tonga-Fiji events (Fig. 2a), the anomalies of $d t^{m-n}$ relative to PREM are observed more or less uniformly across the entire European array (KH2013). Except for the uniform delays, no systematic trends in the differential times with azimuth from the Tonga-Fiji events, which may be amenable to elaborate modeling, have been identified for the high quality events. This would suggest only minor influences of CMB structure on individual paths.

\section{$V_{p}$ model for the outermost core $\tau-p$ inversion: effects of starting model}

KH2013 built a $V_{p}$ model of the topmost $700 \mathrm{~km}$ of the outer core using differential SmKS travel time anomalies of several Fiji-Tonga events recorded at Europe, $d t^{3-2}$, $d t^{4-3}$, and $d t^{5-3}$, to which a $\tau-p$ inversion method had been applied (Garmany et al. 1979; HK2010; KH2013), with PREM as the starting model. The resultant model, KHOMC, has a $0.45 \%$ slower $V_{p}$ than PREM at the CMB 
Table 2 Differential travel times are measured on the waveforms which are obtained by linearly stacking the observed broadband seismograms with the relative slowness computed for PREM

\begin{tabular}{|c|c|c|c|c|c|c|c|c|c|}
\hline Event & Array & Distance & $d t^{3-2}(\mathrm{~s})$ & Bias & $d t^{4-3}(s)$ & Bias & $d t^{5-3}(\mathrm{~s})$ & Bias & Category \\
\hline FEV1 & EU & 154.0 & $1.2 \pm 0.2$ & 0.1 & $1.0 \pm 0.2$ & 0.2 & $1.2 \pm 0.3$ & 0.2 & A \\
\hline FEV2 & EU & 154.0 & $1.1 \pm 0.2$ & 0.2 & $1.2 \pm 0.2$ & 0.2 & $1.2 \pm 0.3$ & 0.3 & A \\
\hline FEV3 & EU & 152.5 & $1.1 \pm 0.3$ & 0.1 & $1.3 \pm 0.3$ & 0.3 & $1.5 \pm 0.4$ & 0.2 & A \\
\hline FEV4 & EU & 152. & $1.6 \pm 0.2$ & 0.2 & $1.3 \pm 0.2$ & 0.2 & $1.2 \pm 0.3$ & 0.3 & A \\
\hline FEV5 & EU & 147.3 & $1.5 \pm 0.3$ & 0.3 & $1.3 \pm 0.4$ & 0.2 & $1.6 \pm 0.5$ & 0.0 & A \\
\hline FEV6 & EU & 153.5 & $1.6 \pm 0.4$ & 0.2 & $0.9 \pm 0.2$ & 0.2 & $1.1 \pm 0.4$ & 0.1 & A \\
\hline FEV6 & US & 123.5 & $0.9 \pm 0.3$ & 0.3 & - & & - & & B \\
\hline FEV7 & EU & 148.5 & $1.5 \pm 0.4$ & 0.3 & $1.4 \pm 0.3$ & 0.4 & $1.3 \pm 0.4$ & 0.4 & B \\
\hline FEV8 & EU & 148. & $1.1 \pm 0.5$ & 0.3 & - & & - & & B \\
\hline FEV9 & EU & 153. & $1.2 \pm 0.5$ & -0.1 & - & & - & & B \\
\hline FEV10 & EU & 151.9 & $0.9 \pm 0.4$ & -0.3 & - & & - & & B \\
\hline FEV11 & EU & 151.9 & $1.1 \pm 0.4$ & 0.0 & - & & - & & B \\
\hline FEV12 & EU & 150.0 & $1.3 \pm 0.2$ & 0.3 & $1.2 \pm 0.2$ & 0.4 & $1.1 \pm 0.3$ & 0.3 & A \\
\hline FEV13 & AF & 131. & $0.2 \pm 0.3$ & -0.1 & $0.2 \pm 0.2$ & -0.1 & - & & B \\
\hline KEV1 & EU & 155. & $1.2 \pm 0.2$ & 0.3 & $1.2 \pm 0.3$ & 0.4 & $1.0 \pm 0.4$ & 0.4 & A \\
\hline KEV2 & EU & 153. & $1.9 \pm 0.4$ & 0.2 & $1.0 \pm 0.3$ & 0.2 & - & & B \\
\hline KEV3 & EU & 155. & $1.4 \pm 0.4$ & 0.2 & - & & - & & B \\
\hline KEV4 & EU & 155. & $1.6 \pm 0.5$ & 0.4 & $1.5 \pm-$ & 0.4 & - & & B \\
\hline KEV5 & EU & 155.5 & $1.2 \pm 0.4$ & 0.3 & $1.2 \pm 0.4$ & 0.2 & $1.5 \pm 0.5$ & 0.1 & A \\
\hline KEV6 & EU & 155. & $1.4 \pm 0.3$ & 0.2 & $1.5 \pm 0.5$ & 0.3 & - & & B \\
\hline AEV1 & $J P$ & 162.4 & $0.5 \pm 0.4$ & 0.1 & $0.9 \pm 0.4$ & 0.2 & - & & B \\
\hline AEV2 & $\mathrm{HI}$ & 157.8 & $0.9 \pm 0.4$ & -0.1 & $1.3 \pm 0.2$ & 0.2 & $1.4 \pm 0.3$ & -0.1 & A \\
\hline AEV3 & $\mathrm{HI}$ & 162.6 & $0.5 \pm 0.5$ & 0.0 & $0.9 \pm 0.4$ & 0.2 & $0.8 \pm 0.5$ & 0.2 & B \\
\hline AEV4 & $\mathrm{HI}$ & 160.0 & $0.6 \pm 0.3$ & -0.1 & $0.6 \pm 0.3$ & 0.3 & $0.5 \pm 0.3$ & 0.2 & A \\
\hline AEV4 & $\mathrm{AU}$ & 124.8 & $0.7 \pm 0.3$ & 0.6 & - & & - & & B \\
\hline AEV4 & AS & 167.9 & $1.0 \pm 0.4$ & 0.0 & - & & - & & B \\
\hline AEV5 & $J P$ & 163.4 & $1.0 \pm 0.4$ & 0.0 & - & & - & & B \\
\hline AEV5 & $\mathrm{AU}$ & 124.7 & $0.6 \pm 0.4$ & 0.4 & - & & - & & B \\
\hline AEV5 & AS & 167.8 & $1.0 \pm 0.4$ & 0.0 & $1.4 \pm 0.5$ & 0.6 & - & & B \\
\hline AEV6 & $J P$ & 153.9 & $0.6 \pm 0.4$ & -0.1 & - & & - & & B \\
\hline NCEV & $A U$ & 140.1 & $2.0 \pm 0.5$ & 0.7 & $1.0 \pm 0.5$ & 0.3 & - & & B \\
\hline IEV1 & US & 130.9 & $1.3 \pm 0.4$ & 0.2 & - & & - & & B \\
\hline IEV2 & EU & 122.8 & $0.9 \pm 0.4$ & 0.1 & - & & - & & B \\
\hline IEV2 & US & 119.5 & $0.9 \pm 0.4$ & 0.1 & - & & - & & B \\
\hline IEV3 & US & 120.9 & $0.8 \pm 0.5$ & -0.4 & - & & - & & B \\
\hline IEV4 & US & 144. & $1.0 \pm 0.4$ & 0.4 & $1.0 \pm 0.5$ & 0.4 & $0.9 \pm 0.5$ & 0.5 & A \\
\hline IEV5 & US & 141. & $0.9 \pm 0.3$ & 0.1 & $0.3 \pm 0.3$ & 0.0 & $0.3 \pm 0.5$ & 0.2 & A \\
\hline IEV6 & US & 133.1 & $0.4 \pm 0.2$ & 0.2 & $0.1 \pm 0.3$ & -0.2 & - & & B \\
\hline MEV & US & 149.2 & $1.0 \pm 0.5$ & 0.2 & - & & - & & B \\
\hline INEV & US & 168.6 & $0.2 \pm 0.5$ & -0.3 & $1.2 \pm 0.4$ & 0.5 & - & & B \\
\hline SEV1 & AK & 154.1 & $0.4 \pm 0.5$ & 0.1 & - & & - & & B \\
\hline SEV1 & $J P$ & 153.8 & $0.9 \pm 0.3$ & 0.2 & - & & - & & A \\
\hline PEV1 & SA & 149.5 & $1.9 \pm 0.3$ & 0.5 & $0.5 \pm 0.5$ & 0.6 & - & & B \\
\hline
\end{tabular}

Array distances correspond to approximate array centers, whose accurate value do not affect the results. The values of "Bias" are measured by applying the array method on the reflectivity seismograms computed for PREM and comparing the differential SmKS travel times with the ray theoretical predictions 
(Fig. 3). The lower-than PREM $V_{p}$ values persist down to about $300 \mathrm{~km}$ below the CMB. We note here that the $\tau-p$ method is a linear inversion that requires a good starting model. Although PREM is known to be a good global model, there may be other reference models as good as PREM. We perform the $\tau-p$ inversion using IASP91 as the starting model, as did Alexandrakis and Eaton (2010). By using only the Fiji-Tonga to European stations data, as in the case of KHOMC, we obtained a profile of $V_{p}$ anomaly relative to IASP91 (called KOCTI). KOCTI has faster $V_{p}$ values than IASP91 in the shallowest 300 $\mathrm{km}$ of the outer core (Fig. 3). KOCTI shows an improved fit to the data compared to IASP91 (Fig. 3), but its overall fit is less suitable than that of KHOMC, with the most significant disagreement for the $d t^{3-2}$ data of larger distances, as will be shown later in this study. The $V_{p}$ values of KOCTI for the upper $300 \mathrm{~km}$ are only about $0.01 \mathrm{~km} / \mathrm{s}$ slower than those of KHOMC (the $V_{p}$ at the $\mathrm{CMB}$ is $-0.045 \mathrm{~km} / \mathrm{s}$ relative to PREM, while that of KHOMC is $-0.035 \mathrm{~km} / \mathrm{s}$ ). Therefore, it can be seen that the two models agree with each other within the uncertainty range (Fig. 3). We conclude that the $V_{p}$ at CMB is constrained well by the $\tau-p$ inversion to $8.03 \pm 0.01 \mathrm{~km} / \mathrm{s}$, irrespective of the starting model. The disagreement between KOCTI and $\mathrm{KHOMC}$ for the depth range from about $300 \mathrm{~km}$ to $700 \mathrm{~km}$ from the $\mathrm{CMB}$ is larger (by about -0.02 $\mathrm{km} / \mathrm{s}$ ), suggesting a poorer resolution than the shallower core.

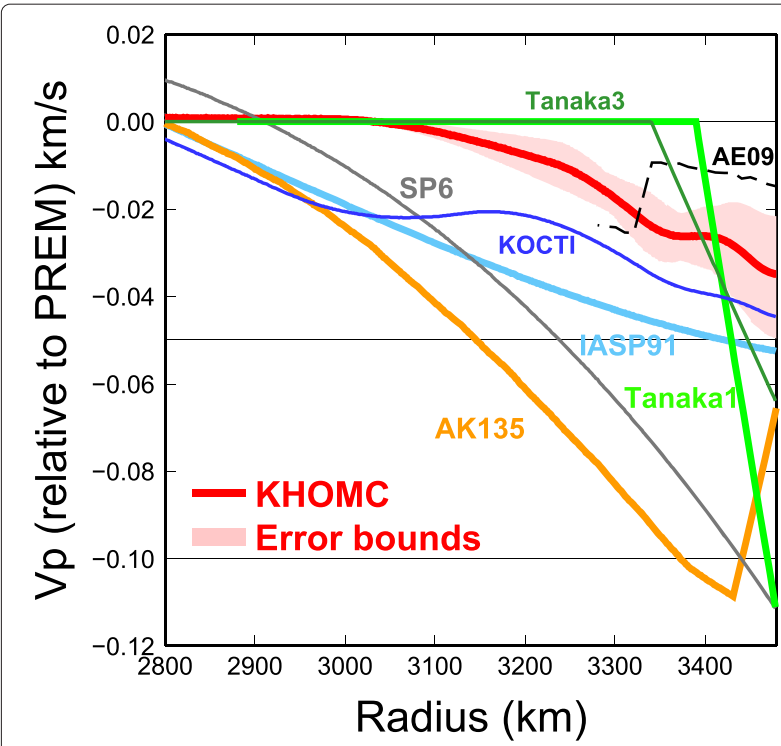

Fig. $3 V_{p}$ models of outermost core. $V_{p}$ models of the upper $700 \mathrm{~km}$ of the outer core: KHOMC, AK135, SP6, Tanaka-1, Tanaka-3, IASP91, AE09 (Alexandrakis and Eaton 2010), and KOCTI. The $V_{p}$ values (in $\mathrm{km} / \mathrm{s}$ ) as a function of depth relative to the PREM values are shown. The uncertainty range of KHOMC are shown in pink (KH2013)

\section{Genetic algorithms}

In order to check further the effects of the starting model on the result, we perform another $V_{p}$ inversion using genetic algorithms (e.g., Yamanaka and Ishida 1995) and attempt to find the global minimum of $d t^{m-n}$ misfit in a fashion that does not explicitly require a starting model. The $V_{p}$ profile of the outer $700 \mathrm{~km}$ of the core is assumed to be continuous and consist of four layers with constant $V_{p}$ gradients. For each layer, the $V_{p}$ value at its top and its thickness are determined (see the caption of Fig. 4 for details).

We use the data set consisting of $d t^{3-2}, d t^{4-3}$, and $d t^{5-4}$ data for four events from Fiji-Tonga to Europe (FEV1, FEV2, FEV3, FEV4) and three events from Argentina to

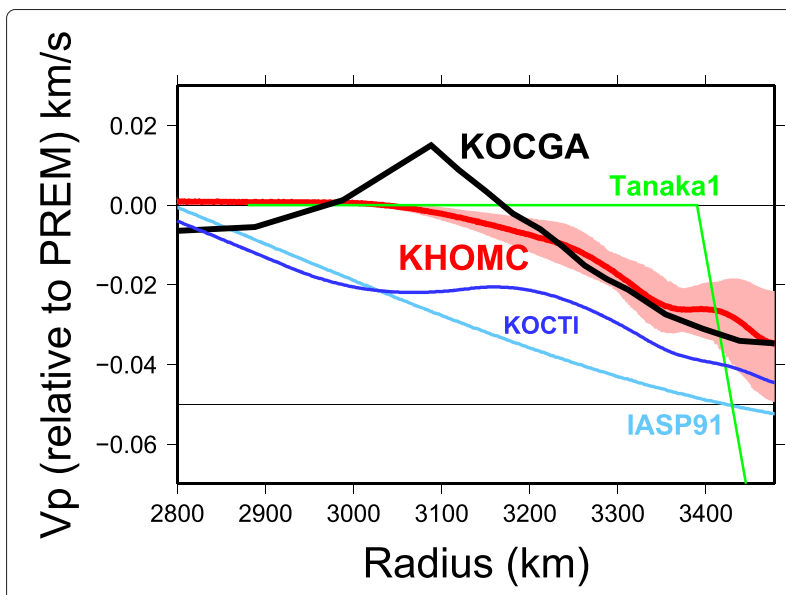

Fig. 4 Genetic algorithm. The $V_{p}$ model obtained by the inversion using genetic algorithms. The thick black line labeled KOCGA is the obtained continuous $V_{p}$ model of the outer $700 \mathrm{~km}$ of the core, which has four layers with uniform $V_{p}$ gradients. Other details are the same as Fig. 3. The thickness of the deepest layer is fixed to $400 \mathrm{~km}$ and the $V_{p}$ value at the bottom of the layer is fixed to that of PREM, so that there are seven parameters to be determined, each of which is described as a 6-bit binary number. Therefore, each gene has a length of 42 bits. The least bit for the $V_{p}$ at the top of each layer corresponds to $0.003 \mathrm{~km} / \mathrm{s}$, while the least bit for the thickness corresponds to 2 $\mathrm{km}$. The depth of the CMB is fixed to that of PREM, so the inversion is not entirely free from the reference model, but the effect is quite small insofar as the differential travel times of SmKS waves are concerned. The range of the $V_{p}$ value sought by this parameterization covers about $\pm 0.2 \mathrm{~km} / \mathrm{s}$ relative to PREM, which is wide enough not to miss any successful models. First, the samples of $V_{p}$ model of the first generation are constructed by randomly selecting the seven model parameters. Second, the misfit of the model predictions to the observations is computed, and each $V_{p}$ model sample is selected with a probability proportional to the inverse of the squared sum of its misfit. After the selection, crossover of the genes between two randomly selected samples occurs with a given probability, followed by the mutation within each gene that occurs with a given probability, in order to generate the samples of the next generation. This process is iterated through the fixed number of generations. The total misfit over the entire samples usually converges rapidly enough. The parameters used in the inversion are as follows: both the mutation probability and the crossover probability are 0.2 , and the numbers of sample and generation are 100 and 40 , respectively 
Japan (AEV1, AEV2, AEV3) without correcting for bias (Table 2). The inversion is repeated 40 times with different initial values, and the case with the least misfit, for which the total residual decreases from 0.5 to $0.15 \mathrm{~s}$ through the 40 generations, is chosen. As shown in Fig. 4, the obtained model (called "KOCGA" hereafter) is fairly close to KHOMC. The agreement between the model and KHOMC is remarkable especially for the shallower 300 $\mathrm{km}$ of modeled depth range. Based on this result, we conclude that the validity of KHOMC is not affected by the choice of the starting model.

\section{SmKS slowness measurements}

In addition to travel time differences, differences in slowness between two SmKS waves provide information on the relative arrival directions of the waves. In HK2010 and $\mathrm{KH} 2013$, it was reported that the relative slownesses for the Tonga and Argentina events are close to those predicted by PREM. This observation has been exploited in using the $\tau-\mathrm{p}$ inversion. The slowness observations can also give constraints on the degree of large-scale heterogeneity in the receiver-side mantle, as shall be shown in the "Discussion" section. The differential slowness between two different S $m K S$ waves changes gradually with distance. Therefore, the moveout of a SmSK wave, relative to the reference S $m K S$ wave on a record section, is aligned on a slightly curved line rather than a straight line. Although the curvature is small, when the aperture of an array is as large as $20^{\circ}$, its effect on the differential travel times approaches a second, which could give rise to a small bias of the measured differential slowness between

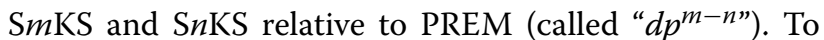
ensure that $d p^{m-n}$ is measured accurately, we correct for the curvature by assuming that the moveout around the array center (distance of $\Delta_{0}$ ) is a quadratic function of epicentral distance when stacking seismograms. Accordingly, the differential slowness between S3KS and S2KS (and similarly between S4KS and S2KS) is expressed as

$$
d p^{3-2}(\Delta)=d p^{3-2}\left(\Delta_{0}\right)+\left(\Delta-\Delta_{0}\right) \frac{d}{d \Delta} d p^{3-2}
$$

The curvatures of the moveout curve are fixed to those of the reference model, so that $\frac{d}{d \Delta} d p$ is 0.014 and 0.021 $\mathrm{s} /{ }^{2}$ for $d p^{3-2}$ and $d p^{4-2}$, respectively, which does not significantly affect the results. We obtain precise slowness measurements of S3KS and S4KS waves with S2KS as the reference wave for eight Fiji-Tonga and Kermadec events observed from Europe, and four Argentina events observed from Japan (Table 3). The $d p^{3-2}$ and $d p^{4-2}$ are then compared to the predictions of KHOMC (Table 3).

Figure 5a shows a vespagram example of the cube-root stack output as a function of slowness and arrival time relative to S2KS, with the small moveout curvature effects corrected. The observed $d p^{3-2}$ and $d p^{4-2}$ (red circles) closely agree with theoretical predictions by KHOMC (Table 3), which are shown by red stars in the figure. The RMS residuals computed for the Fiji-Tonga and Argentina events using the outer core models (PREM, IASP91, KOCTI, AK135, SP6, and Tanaka-1) show that the least residual is obtained for KHOMC (Fig. 5b). The RMS residual is less than $0.03 \mathrm{~s} /{ }^{\circ}$ for KHOMC (Fig. 5b), and $d p^{3-2}$ for the majority of the events, which agree with KHOMC within $0.02 \mathrm{~s} /{ }^{\circ}$ (Table 3). The residuals for KHOMC of $d p^{3-2}$ and $d p^{4-2}$ are slightly improved in comparison to PREM, while no such $d p^{m-n}$ improvement is seen for the other models. The small discrepancy of the observed $d p^{3-2}$ and $d p^{4-2}$, with respect to PREM, justifies the usage of PREM as the reference model for stripping the mantle contributions from the observed distance ranges and travel times in the $\tau$-p inversion that resulted in KHOMC (HK2010; KH2013). While the primary information about the core $V_{p}$ structure is contained in the differential SmKS travel times, as shown in parameter tests (KH2013), the measured $d p^{3-2}$ and $d p^{4-2}$ data not only indicate the validity of our travel time analyses but also further support the validity of KHOMC.

\section{SmKS differential times of other regions Observations of $\boldsymbol{d t}^{3-2}$}

KHOMC was built by using the Tonga-Fiji data set that samples rather limited areas of the CMB, mostly beneath western Pacific and Siberia. KH2013 showed that the SmKS differential travel times of the Argentina to Japan data set are consistent with KHOMC, even though they sample entirely different parts of the CMB. In this study, we compile the $d t^{3-2}$ measurements for additional pairs of event and large-scale array that sample a much larger portion of the CMB. We determine the anomalies of $d t^{3-2}$ for several large-scale arrays (Japan, US, Australia, Alaska, Africa, South America, and Southeast Asia; Fig. 6a) using the same processing. The ray paths cover a reasonably large part of the outer core (Fig. 6a).

A striking feature of the result is that the $d t^{3-2}$ observations based on the large-scale array processing are quite well matched by KHOMC (Fig. 6b). On the other hand, the $d t^{3-2}$ values measured for individual stations (Souriau et al. 2003) show a much larger scatter than the array measurements (Fig. 6b), which suggests that measuring $d t^{3-2}$ using large-scale array data significantly ameliorates the heterogeneous mantle structure effects. Another notable feature is that the array measurements fall approximately in the middle of the range of individual station measurements (Figure six b; Souriau et al. 2003), which also suggests that the array measurement of this study has successfully represented the overall core structure. The total residual of the array measurements is computed for KHOMC and for other outer core $V_{p}$ models (IASP91, Tanaka-1, Tanaka-3, ak135, SP6, and PREM), and the least 
Table 3 Differential slowness $\left(d p^{3-2}\right.$ and $\left.d p^{4-2}, s^{\circ}\right)$

\begin{tabular}{|c|c|c|c|c|c|c|c|}
\hline Event & Distance $\Delta_{0}$ & $d p^{3-2}\left(s /{ }^{\circ}\right)$ & KHOMC & Residual & $d p^{4-2}\left(s /{ }^{\circ}\right)$ & KHOMC & Residual \\
\hline FEV1 & 154.0 & 1.16 & 1.16 & 0.0 & 1.62 & 1.61 & 0.01 \\
\hline FEV2 & 154.0 & 1.20 & 1.16 & 0.04 & 1.65 & 1.61 & 0.04 \\
\hline FEV3 & 152.5 & 1.15 & 1.13 & 0.02 & 1.60 & 1.57 & 0.03 \\
\hline FEV4 & 152.0 & 1.14 & 1.13 & 0.01 & 1.59 & 1.57 & 0.02 \\
\hline FEV6 & 153.5 & 1.19 & 1.18 & 0.01 & 1.65 & 1.65 & 0.00 \\
\hline FEV12 & 150.0 & 1.12 & 1.11 & 0.01 & 1.56 & 1.53 & 0.03 \\
\hline KEV1 & 155.0 & 1.20 & 1.16 & 0.04 & 1.64 & 1.62 & 0.02 \\
\hline AEV2 & 157.8 & 1.19 & 1.21 & -0.02 & 1.70 & 1.69 & 0.01 \\
\hline AEV3 & 162.6 & 1.20 & 1.18 & 0.02 & 1.65 & 1.65 & 0.00 \\
\hline AEV4 & 160.0 & 1.30 & 1.23 & 0.07 & 1.77 & 1.73 & 0.04 \\
\hline AEV5 & 167.8 & 1.30 & 1.28 & 0.02 & 1.80 & 1.77 & 0.02 \\
\hline
\end{tabular}

The corresponding values calculated for KHOMC and the residuals of the observed values from the KHOMC predictions are also shown. Alignment is at the center distance of the array

misfit is obtained for KHOMC (Fig. 6c). As mentioned previously, we note that KHOMC definitely gives a better fit than the $\tau$-p model based on IASP91 (KOCTI) for larger distances. These observations indicate that the mantle effects on $d t^{3-2}$ are of secondary importance and that the essential features of KHOMC relative to PREM reflect the structure of the core.

\section{Observations of $d t^{4-3}$ and $d t^{5-4}$}

The ray paths for which $d t^{4-3}$ can be determined by using large-scale array data are geographically more restricted around Pacific than in the case of $d t^{3-2}$ but still sample a reasonably broad portion of the CMB (Fig. 7a). The measured $d t^{4-3}$ values fall in the middle of individual station measurements (Garnero et al. 1993) and are well consistent with KHOMC (Fig. 7b). Among the $V_{p}$ models considered (Fig. 7d), the $d t^{4-3}$ observations are best matched by KOCTI, but the data set (especially for category A) is explained almost equally well by KHOMC; the $d t^{4-3}$ data set is not useful for discriminating between the two models. The other models (IASP91, Tanaka-1, Tanaka-3, AK135, and SP6) give significantly poorer fits when compared with KHOMC and KOCTI.

The $d t^{5-4}$ data set is matched well by some of the models considered, KHOMC, PREM, IASP91, and KOCTI (Fig. 7c), so that it is not crucial to discriminate between the models. Nevertheless, it clearly refutes the class of models that have a strong $V_{p}$ reduction in a thin layer at the top of the outer core, such as Tanaka-1, Tanaka-3, and that of Garnero et al. (1993). These models were built without using $d t^{5-4}$ and were aimed to match mainly $d t^{3-2}$, which means that the amount of $V_{p}$ anomaly relative to PREM across the top several hundred kilometers of the outer core can be well predicted by these models. The mismatch between these models with the observed $d t^{5-4}$ means that the $V_{p}$ anomaly relative to PREM needs to be distributed over a broader depth range than those in the models. This also indicates that the $V_{p}$ gradient near the top of the core is not extremely anomalous compared to PREM (Fig. 3). The inference is further supported by the good match of the observed S6KS waveforms relative to S5KS by KHOMC (KH2013); the $V_{p}$ gradient near the top of the core is tightly constrained by our data set.

We emphasize again that KHOMC was constructed by using the Fiji-Tonga to Europe data set alone, yet the observations of $d t^{4-3}$ and $d t^{5-4}$ for different regions can be matched by the same model quite well. The SmKS data we used, therefore, should primarily reflect the outer core structure.

\section{Anomalous outermost core in terms of $V_{p}$ gradient}

The most important feature of our $V_{p}$ models (KHOMC and KOCGA) is the presence of marked radial change in the $V_{p}$ gradient, $V_{p}^{\prime}=d V p / d r$; the outermost core of KHOMC and KOCGA is essentially characterized by two distinctive layers with different $V_{p}^{\prime}$. We parameterize the $V_{p}$ structure of the outermost $700 \mathrm{~km}$ of the core with two layers that have constant $V_{p}^{\prime}$ and compute the misfits of $d t^{3-2}, d t^{4-3}$, and $d t^{5-4}$ for the Fiji-Tonga and Argentina data sets (KH2013).

The minimum misfit in this modeling is obtained for the $V_{p}$ value at the CMB near that of KHOMC $(-0.35 \mathrm{~km} / \mathrm{s}$ with respect to PREM). For this case, the obtained $V_{p}^{\prime}$ value of the shallower layer approximately agrees with the mean values of $V_{p}^{\prime}$ of the shallower $300 \mathrm{~km}$ for KHOMC and KOCGA (Fig. 8), which indicates that the mean $V_{p}$ gradient of the shallower layer is well constrained (Fig. 8) by the presence of additional different $\mathrm{S} m \mathrm{KS}$ waves passing through it. Although the lower layer gradient is rather poorly constrained, the significant difference in the values of $V_{p}^{\prime}$ anomalies relative to PREM between the two layers is robust; the anomaly of $V_{p}^{\prime}$ with respect to PREM 

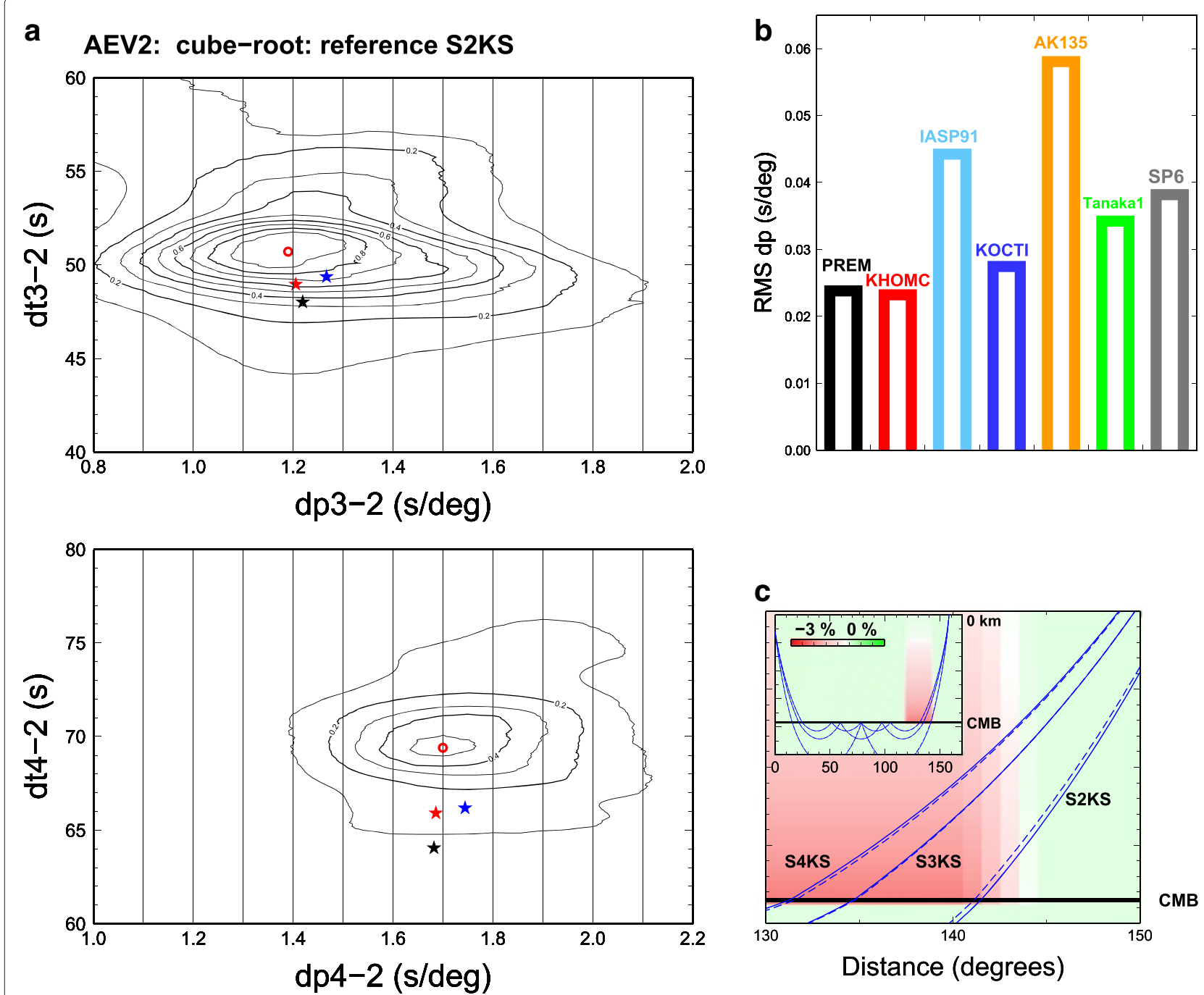

Fig. 5 Slowness measurements. (a) The observed differential slownesses, $d p^{3-2}$ (top) and $d p^{4-2}$ (bottom). Horizontal axis is slowness (in $s /{ }^{\circ}$ ) as a function of time after the arrival of S2KS (vertical axis). Red, blue, and black stars indicate the predictions from KHOMC, IASP91, and PREM, respectively. (b ) RMS misfits of $d p^{3-2}$ and $d p^{4-2}$ in $s /{ }^{\circ}$ for different outer core models. The difference in the misfit between Takana- 1 and Tanaka-3 is small. (c) An artificial model of 2D V heterogeneity localized near the bottom of the mantle receiver-side structure and the ray paths of S2KS, S3KS, and S4KS, after the Earth-flattening transformation. Dashed and solid blue rays are those for the unperturbed and perturbed Earth models, respectively. Inset shows the entire paths, while the rays near the receiver side CMB are focused below. The $V_{s}$ anomaly is shown with color scale

$\left(d V_{p}^{\prime}=d V p / d r_{\text {profile }}-d V p / d r_{P R E M}\right)$ is much larger in the shallower part of the depth range considered than below it (Fig. 8). Accordingly, the $V_{p}^{\prime}$ of the shallower $300 \mathrm{~km}$ of the outer core is steeper than PREM by about $0.0001(1 / \mathrm{s})$, while that of the deeper part is closer to that of PREM. The number anomalies of $V_{p}^{\prime}$ (relative to PREM) obtained for the Genetic algorithms (KOCGA) is $0.00012(1 / \mathrm{s})$ for the upper $\sim 300 \mathrm{~km}$ of the core, while that is $-0.00002(1 / \mathrm{s})$ for the deeper part (300 to $700 \mathrm{~km}$ from the CMB).

The $V_{p}$ gradient and the pressure derivative of bulk modulus $\left(K^{\prime}=d K_{s} / d P\right)$ are interrelated, and the principal feature of KHOMC indicates a substantial radial variation in $K^{\prime}$ within the uppermost $700 \mathrm{~km}$ of the outer core. By using the equation, $V_{p}^{\prime}=-g\left(2 V_{p}\right)^{-1}\left(K^{\prime}-1\right), K^{\prime}$ can be computed from $V_{p}$. We find that the $K^{\prime}$ value of the outermost $300 \mathrm{~km}$ of the core is nearly 3.7 , which is larger than that of the deeper core by about 0.2 (Butler and Anderson 1978). The estimated anomaly of $K^{\prime}$ for the upper 300 to $400 \mathrm{~km}$ of the outer core amounts to a nearly $5 \%$ radial anomaly, which is more than an order of magnitude larger than the $V_{p}$ anomaly itself.

\section{Discussion}

\section{Effects of receiver-side mantle}

Our previous studies have shown that large-scale array analyses adequately extract differential SmKS travel time 

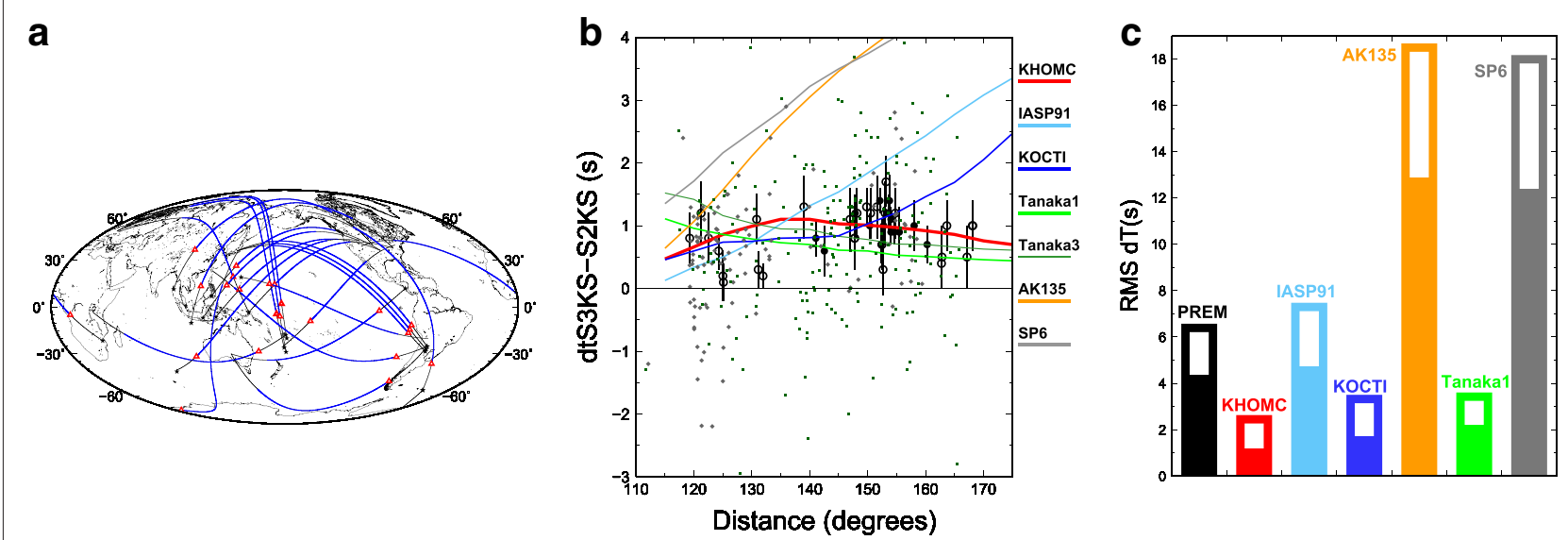

Fig. 6 Globally measured $d t^{3-2}$ values. (a) Ray paths corresponding to the measured $d t^{3-2}$. Stars and red triangles show the epicenters and source side CMB entry points of S3KS. Blue portions of the ray paths indicate the segments within the outer core. For the events at Tonga-Fiji and Kermadec, only selected events and ray paths are plotted in order to prevent the figure from becoming excessively cluttered. (b) Measured $d t^{3-2}$ for other large-scale arrays converted to a hypothetical focal depth of $500 \mathrm{~km}$. The $d t^{3-2}$ values predicted by global outer core models are shown with lines. The measured $\mathrm{dt}^{3-2}$ are shown by circles with error bars. Filled symbols show the data of category A, while open symbols are of category B (Table 2). The observed $d t^{3-2}$ values are explained quite well by KHOMC (red line). Superimposed are the individual station-based $d t^{3-2}$ data converted to $500 \mathrm{~km}$ focal depth (large diamonds) (courtesy of A. Souriau). Smaller squares are other $\mathrm{dt}^{3-2}$ measurements made for individual stations taken from Souriau et al. (2003) without depth corrections. (c) Total residuals, defined as the square root of the squared sum of the residuals of $d t^{3-2}$ for different outer core models. Filled portions are the misfits for the category A data alone. The colors used for the models correspond to those in (b)

anomalies representative to the array as a whole (HK2010; KH2013). The receiver-side CMB piercing points for the Tonga-Fiji events scatter widely beneath Europe (HK2010; KH2013), making it difficult to envisage a receiverside mantle heterogeneity which causes a systematic anomaly of SmKS differential travel times across the entire array.

Nevertheless, we attempt to conservatively evaluate the effects of mantle heterogeneity on the $d t^{3-2}$ and $d t^{4-3}$ measurements, by focusing on the observation of very

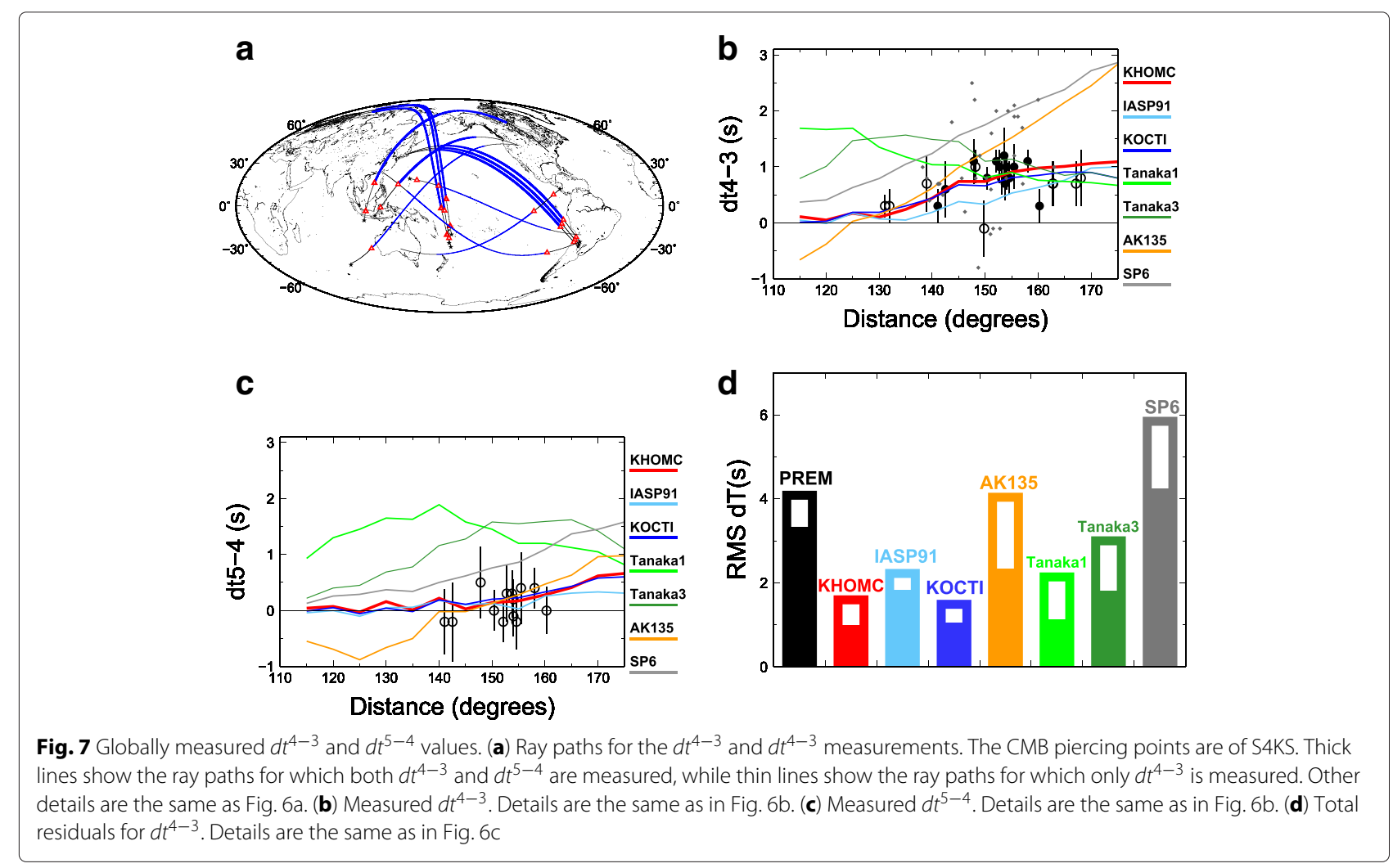




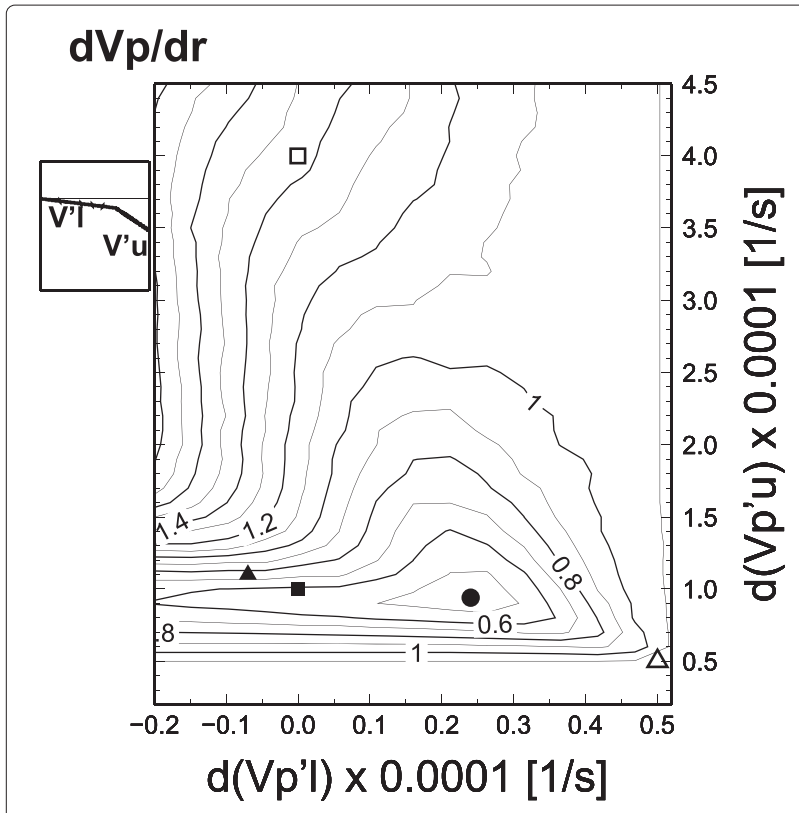

Fig. $8 V_{p}$ gradient with respect to PREM for a two-layer outermost core. Contour plot of total residual (in seconds) of $d t^{3-2}, d t^{4-3}$, and $d t^{5-3}$ for the two-layered outer core model for the case of $V_{p}$ at the CMB of $8.03 \mathrm{~km} / \mathrm{s}$, which give the least residual. The input data are as follows: $d t^{3-2}=1.0, d t^{4-3}=1.0$, and $d t^{5-3}=0.9$ for $154^{\circ}$ and $\mathrm{h}=$ $500 \mathrm{~km}$ representing typical values of the Fiji-Tonga data set; and $d t^{3-2}=0.7, d t^{4-3}=0.9$, and $d t^{5-3}=0.9,162^{\circ}$ and $\mathrm{h}=590 \mathrm{~km}$, representative of the Argentina data set. The deviation of $V_{p}^{\prime}$ from PREM ( $\left.d V p / d r_{\text {profile }}-d V p / d r_{P R E M}\right)$ for the shallower part of the depth range (vertical axis) and the that of the deeper layer (horizontal axis). The points approximately representing KHOMC, KOCGA, IASP91, and Tanaka-1 are shown by a solid square, solid triangle, open triangle, and open square, respectively

small residuals of differential slownesses $d p^{3-2}$ and $d p^{4-2}$ described above from KHOMC (Fig. 5b). The observations indicate that the relative arrival angles of the different SmKS waves are barely anomalous and the rays of the SmKS waves are not substantially bent with respect to each other. A $d p^{3-2}$ anomaly of $0.02 \mathrm{~s} /{ }^{\circ}$ or less for the majority of the events (Table 3 ) corresponds to the anomaly in the separation of S3KS and S2KS piercing points at the $\mathrm{CMB}$ of the receiver side by less than $5 \mathrm{~km}$.

We argue that a differential travel time anomaly $\left(d t^{3-2}\right.$ and $d t^{4-3}$ ) as large as those observed would need to be accompanied by a large anomaly in the relative direction of ray arrivals at the receivers when the anomaly is caused by the mantle heterogeneity beneath the receiver (Fig. 5c). For a low $V_{s}$ heterogeneity to cause a $d t^{3-2}$ anomaly exceeding $1 \mathrm{~s}$ across the array, S2KS waves would need to more effectively avoid the heterogeneous body compared to S3KS (e.g., Garnero and Helmberger 1995). This effect on the ray angle deviations was evaluated by ray tracing experiments. The typical dominant period of S2KS and S3KS is nearly $10 \mathrm{~s}$, and $d t^{3-2}$ values measured for $3 \mathrm{~s}$ high-pass filtered seismograms of three Tonga-Fiji events (FEV2, FEV3, and FEV4) and two Argentina events (AEV2 and AEV4) agree with those for the original broadband seismograms within $0.3 \mathrm{~s}$. They agree within $0.1 \mathrm{~s}$ for two of the Fiji events. This result confirms the utility of conducting theoretical ray estimations on $d t^{3-2}$ to identify possible mantle heterogeneity effects. Therefore, we will next consider a test case involving an artificially strong and sharp-edged $2 \mathrm{D}$ low- $V_{s}$ anomaly that extends about $1000 \mathrm{~km}$ from the CMB with a $V_{s}$ anomaly that is a maximum $3 \%$ slower in the receiver side of the lowermost mantle (Fig. 5c). The $d t^{3-2}$ and $d t^{4-3}$ anomalies at approximately $150^{\circ}$ are $1.8 \mathrm{~s}$ and $0.8 \mathrm{~s}$, respectively, which are comparable to the observations. The relative slownesses, $d p^{3-2}$ and $d p^{4-2}$, are 0.10 and $0.14 \mathrm{~s} /{ }^{\circ}$, respectively, which are nearly five times larger than the observations. Accordingly, the rays of S3KS and S2KS, as well as those of S4KS and S2KS, bend relatively by approximately $25 \mathrm{~km}$ at the CMB. The observed minute anomalies in $d p^{3-2}$ and $d p^{4-2}$ indicate that the receiver-side piercing points are much less significantly bent than is required by this model. The maximum $V_{s}$ anomaly needs to be as low as $0.6 \%$ in order to match the observed $d p^{3-2}$ and $d p^{4-2}$, which sets the upper bounds on the allowable biases of $d t^{3-2}$ and $d t^{4-3}$ due to the receiver-side heterogeneity to much less than $0.4 \mathrm{~s}$ and $0.2 \mathrm{~s}$, respectively.

\section{Effects of source-side mantle}

A source-side lower mantle structure that is capable of causing a $d t^{3-2}$ anomaly of $\sim 1 \mathrm{~s}$ across the entire European array would need to be laterally much larger than $200 \mathrm{~km}$ (KH2013). For the source-side mantle sampled by the Fiji-Tonga data set, the $V_{s}$ structure in the $\mathrm{D}^{\prime \prime}$ of very large scale $(\geq 3000 \mathrm{~km})$ beneath the north of Vanuatu seems to have been resolved moderately well by global seismic tomography (e.g., Lekic et al. 2012). Therefore, it would appear worthwhile to check whether $V_{s}$ heterogeneity of a larger scale in the source side deep mantle accounts for a significant portion of the SmKS differential travel time anomalies.

We focus on five Fiji-Tonga events that are located from 300 to $1000 \mathrm{~km}$ laterally separately from each other (Fig. 9a) and for which high quality S2KS, S3KS, and S4KS have been observed (Table 2). The five events sample the CMB regions that are shifted systematically by 200 to 600 $\mathrm{km}$; each event covers a CMB area of $\sim 1000 \mathrm{~km}$ by 300 $\mathrm{km}$ (Fig. 9b). Effects of $\mathrm{D}^{\prime \prime}$ should be most significant for $d t^{3-2}$, since S2KS and S3KS have larger CMB piercing point separations; $d t^{4-3}$ should be less sensitive to heterogeneity in $\mathrm{D}^{\prime \prime}$ as the separations of the CMB entry points between S3KS and S4KS are less than half of $d t^{3-2}$.

We find that the $d t^{3-2}$ anomalies for the five events are nearly uniform and are excellently matched by KHOMC (Fig. 10a), which is also the case for $d t^{4-3}$ (Fig. 10b). The 

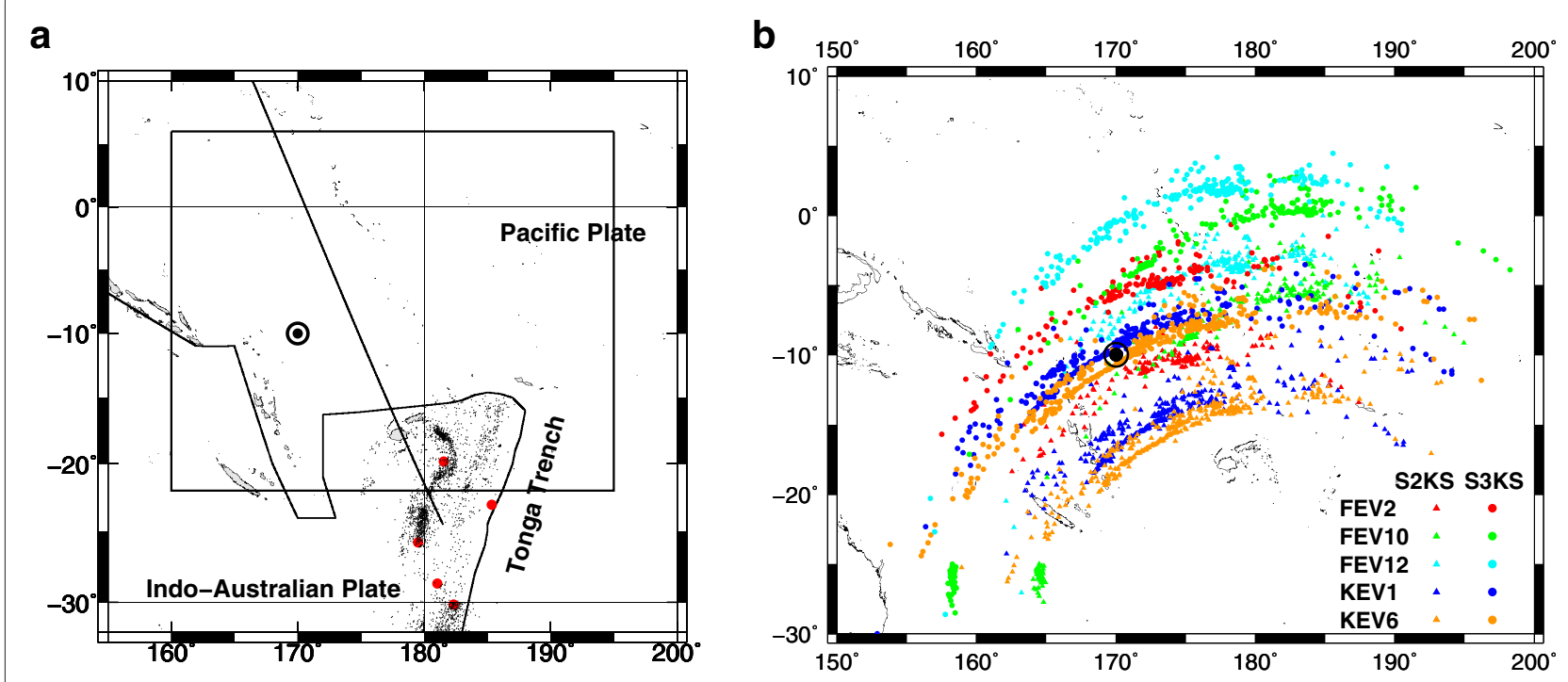

C

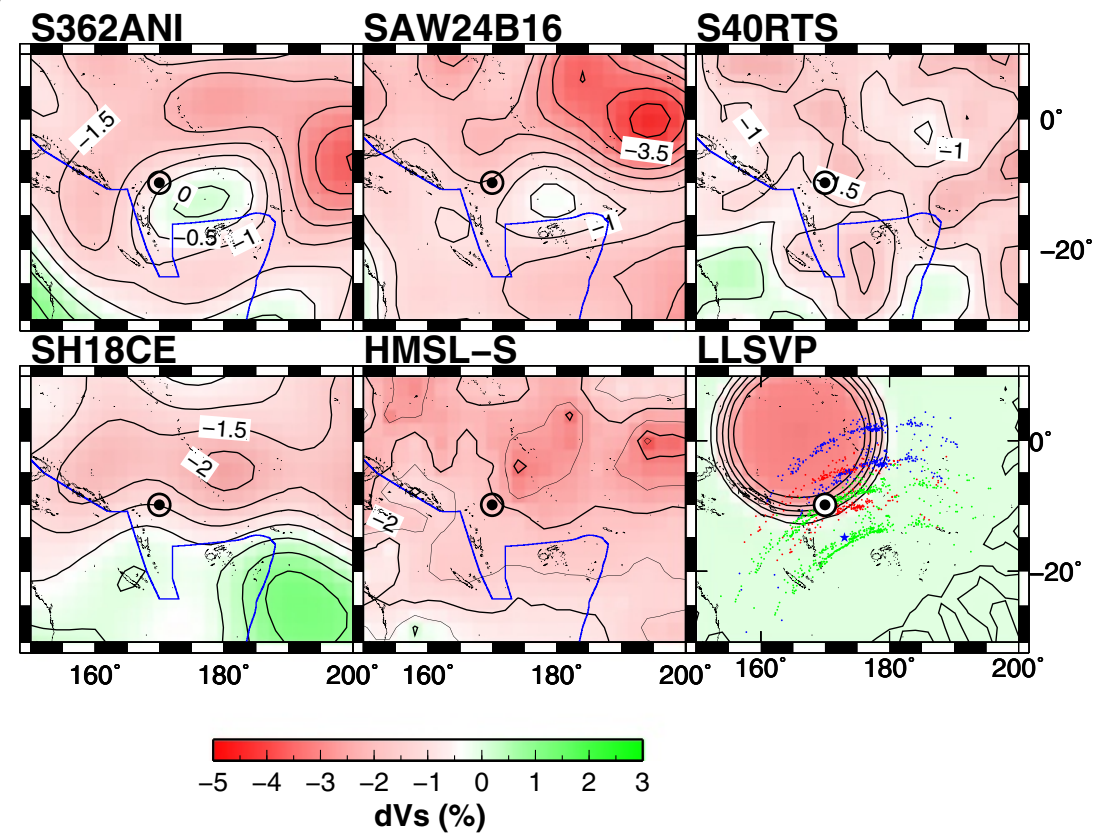

Fig. 9 Tonga-Fiji events. (a) Locations of the five events, FEV2, FEV10, FEV12, KEV1, and KEV6. (b) CMB piercing points for S2KS and S3KS for the four events. Triangles and circles show the core entry points of S2KS and S3KS for each event, respectively. The large black circles are drawn to mark a reference point referred as below. (c) The $V_{s}$ anomalies in the $D^{\prime \prime}$ beneath Vanuatu around the CMB piercing points of the Fiji-Tonga data set for the five tomography models: SAW24B16 (Megnin and Romanowicz 2000), S362ANI (Kustowski et al. 2008), HMSL (Houser et al. 2008), SH18CE (Takeuchi 2007), and S4ORTS (Ritsema et al. 2011). The $V_{s}$ anomalies are shown with color code and contours. The large black circles show the reference point. Bottom right panel shows a model with an extreme and sharp-edged anomaly, like LLSVP. The piercing points of S2KS and S3KS for three of the five events are shown in this panel only

$V_{s}$ models produced by global tomography appear to provide moderately reliable images of the deepest $\sim 200 \mathrm{~km}$ of the mantle (essentially corresponding to $\mathrm{D}^{\prime \prime}$ ) of this region, at least for wavelength features exceeding 2000 $\mathrm{km}$. According to the $V_{s}$ models for the $2800 \mathrm{~km}$ depth of five different tomography studies (Fig. 9c), despite considerable differences in detail, the presence of 1 to $3 \%$ low
$V_{s}$ in the $\mathrm{D}^{\prime \prime}$ of the northern half of the study area seems to be commonly resolved. On the other hand, the depth extent of such anomalies are very poorly constrained, and $2500 \mathrm{~km}$ depth tomography images show only insignificant anomalies that differ substantially from model to model. Therefore, we only deem the heterogeneity in the $\mathrm{D}^{\prime \prime}$ to be significant and evaluate its effects on SmKS travel 

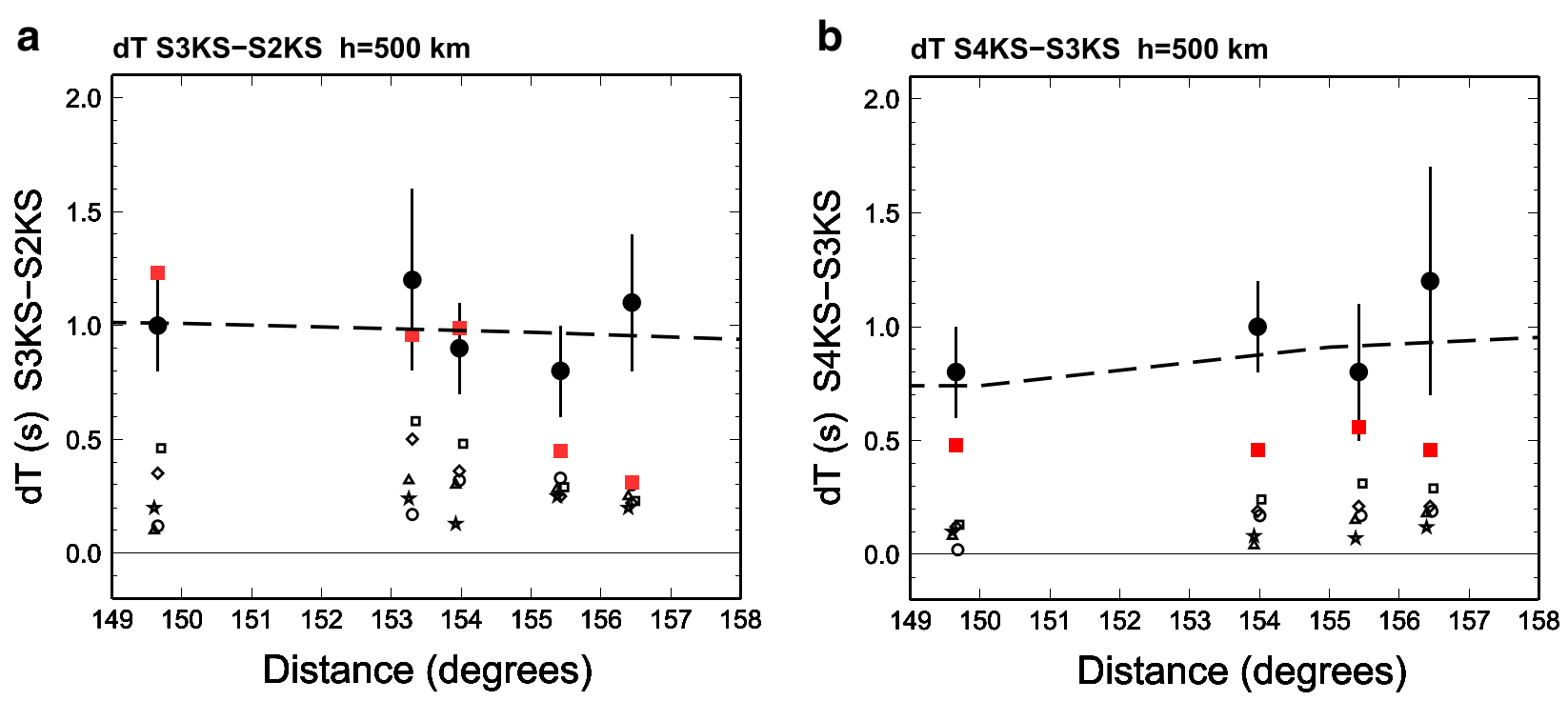

Fig. 10 Effects of source side $D^{\prime \prime}$. (a) Symbols with error bars show the anomalies with respect to PREM of $d t^{3-2}$ measured for the five events in Fig. 9a as a function of array center distance from the epicenters. Small symbols are the predicted effects of the D" heterogeneity for five different tomography models (those in Fig. 9c). Large red squares show the anomalies of $d t^{3-2}$ predicted by the artificial lower mantle heterogeneity model shown in the bottom right panel of Fig. 9c. (b) Same as (a) for $d t^{4-3}$

times, rather than attempting to make corrections on the S $m$ KS travel times for the effects of mantle heterogeneity. Ray theoretical travel times computations show that the tomography-derived structures of $\mathrm{D}^{\prime \prime}$ could systematically affect the anomalies of $d t^{3-2}$ values by $0.3 \mathrm{~s}$ on average (Fig. 10a), while the $d t^{4-3}$ anomalies by $0.2 \mathrm{~s}$ (Fig. 10b). These effects may cause small biases to the core models but do not alter them significantly.

If an extreme but currently unresolved heterogeneity that is analogous to LLSVP with a sharp edge exists near the source side CMB entry points of $\mathrm{S} m \mathrm{KS}$, it might cause $d t^{3-2}$ and $d t^{4-3}$ anomalies of the observed magnitude. As an example of such a scenario, we will next consider a simplified but significantly exaggerated model that has a qualitative resemblance with the tomographic $V_{s}$ anomalies of the $D^{\prime \prime}$ (Fig. 9c) and evaluate the effects of the extreme lower mantle heterogeneity. The model that we will consider has an axisymmetrical tabular-shaped low $V_{s}$ heterogeneity that has a maximum anomaly of $3.5 \%$ at the CMB and that exponentially decays upward with a scale height of $500 \mathrm{~km}$ (Fig. 9c, bottom right). Theoretical $d t^{3-2}$ and $d t^{4-3}$ for this model are computed by ray theory (Fig. 10a, b). The values of $d t^{3-2}$ can be as large as the observed values depending on the epicentral distance. However, there should be a clear trend in $d t^{3-2}$ with the epicentral distance by about $1 \mathrm{~s}$, which is entirely different from the observed $d t^{3-2}$ trend. The relative magnitudes of $d t^{3-2}$ and $d t^{4-3}$ are also grossly inconsistent with the observation. The heterogeneity model significantly underestimates as a $d t^{4-3}$ value that is less than half of the observations, mostly because of the smaller separation of the CMB piercing points (Fig. 10a). Although this demonstrates only just one example, the basic feature of $V_{s}$ structures that potentially cause $d t^{3-2}$ anomalies as large as the observed values should be more or less the same. S2KS more effectively avoids the low $V_{s}$ body than S3KS. Simplified mantle heterogeneity models resembling tomography images, no matter how pronounced and sharp they are, have difficulty matching the observed $d t^{3-2}$ and $d t^{4-3}$ of the Tonga-Fiji data set. Therefore, we conclude that an unresolved lower mantle heterogeneity is unlikely to be the predominant cause of the observed SmKS anomalies, and estimate its effects on $d t^{3-2}$ and $d t^{4-3}$ values based on the current tomography models to be less than $0.3 \mathrm{~s}$ and $0.2 \mathrm{~s}$, respectively.

\section{Interpretations in terms of the composition of the core}

As suggested by high estimates of liquid iron thermal conductivity, a thermally stratified layer in the neighborhood of $100 \mathrm{~km}$ thick might exist in the outermost core (Pozzo et al. 2012). However, a more recent numerical estimate of the electrical conductivity of iron under the Earth's core conditions has negated the thermal stratification requirement (Zhang et al. 2015). If the stratified layer is nearly $300 \mathrm{~km}$ thick, as estimated in this study, the maximum temperature excess over the adiabat at the CMB would be about $300 \mathrm{~K}$. Recent ab initio calculations of liquid and solid iron show that the bulk sound speeds of iron are mostly independent of temperature (Ichikawa et al. 2014; Vocadlo et al. 2009). Therefore, the observed $V_{p}$ and $V_{p}^{\prime}$ anomalies in the uppermost $300 \mathrm{~km}$ of the outer core cannot be of thermal origin and should primarily reflect 
compositional heterogeneity. Since the effective $d K / d P$ in the layer is larger than the bulk of the core, if the light elements diffuse downward from the CMB (and thus have concentrations decreasing with depth), the addition of the light elements must decrease not only the density of the liquid iron alloy but also its bulk modulus. According to recent $\mathrm{ab}$ initio calculations of liquid iron-alloy under the core conditions (Badro et al. 2014), these requirements are satisfied. However, the same calculations show that including light elements increases $V_{p}$. Thus, it seems that matching the observed $V_{p}$ value at the CMB cannot be done by simply by adding light elements, even though the effects of non-ideal mixing in the iron alloy (which might not be adequately modeled in the simulations) might still play a role in reducing $V_{p}$ (Helffrich 2012).

The estimated thickness of the compositionally stratified layer $(\sim 300 \mathrm{~km})$ cannot be interpreted via a straightforward process. If the stratified layer evolved from the $\mathrm{CMB}$ through the diffusion of light elements, the thickness of the layer is essentially determined by the diffusion coefficient of the core liquid. The mass diffusivity of liquid iron under core conditions is thought to be reasonably well constrained (Koci et al. 2007; Pozzo et al. 2012; Helffrich 2014), and the expected thickness is no more than $80 \mathrm{~km}$ (Buffett and Seagle 2010; Helffrich and Kaneshima 2013). Helffrich (2014) suggests that the presence of a thick layer is a feature of the Earth's core that was formed at the time of the putative giant impact.

While the $V_{p}$ profile of the top $700 \mathrm{~km}$ of the core is adequately represented by two layers with nearly constant radial $V_{p}$ profile gradients, there is certainly room for the profile to be optimized with some physically plausible constraints, such as the diffusion profile of light elements (Helffrich 2014). However, the revelation of detailed features of the $V_{p}$ profile is somewhat more difficult due to the presence of mantle effects that have secondary importance. Based on the lack of corresponding anomalies in the waveforms, a sharp interface with a large $V_{p}$ jump at the bottom of the shallower layer near the depth of 300 $\mathrm{km}$ is unlikely to exist, but the presence of a weak jump cannot be ruled out. If light elements diffuse from the $\mathrm{CMB}$, and if double diffusion takes place to form the stratified layer, a succession of thin homogeneous layers might occur near the bottom of the stratified layer (e.g., Buffett and Seagle 2010). In such cases, scattering of seismic energy might occur near the bottom of the layer, depending on the contrasts in the elastic properties between the materials enriched and depleted in light elements. A search for such scattering waves might reveal further details about the enigmatic region of deep Earth. On the other hand, the very top of the core is obviously another locality where an anomalous structure is possible. The existence of a thin and anomalously high $V_{p}$ and low density layer at the top of the core (Helffrich and Kaneshima
2004) is not supported, if the layer thickness exceeds $10 \mathrm{~km}$ or so, by a good fit of the waveforms S6KS to KHOMC (KH2013). Nevertheless, a thinner layer might exist.

\section{Conclusions}

The differential travels between SmKS measured by analyzing large-scale broadband seismometer arrays are shown to predominantly reflect the $V_{p}$ structure of the outermost outer core. The combination of $d t^{3-2}, d t^{4-3}$, and $d t^{5-4}$ anomalies restrict permissible $V_{p}$ models within a narrow range. There is a significant radial change in gradient of $V_{p}$ at the depth about $300 \mathrm{~km}$ below the $\mathrm{CMB}$. The gradient of the shallower layer corresponds to an effective change in $d K_{s} / d P$ by about 0.2 , which is too large to be attributed to thermal effects alone, and requires compositional stratification.

\section{Abbreviations}

LLSVP, Large low shear velocity province; HK2010, Helffrich and Kaneshima (2010); KH2013, Kaneshima and Helffrich (2013).

\section{Competing interests}

The authors declare that they have no competing interests.

\section{Authors' contributions}

SK performed the analyses and wrote the manuscript. TM prepared for the Hi-net tilt-meter data. Both authors read and approved the final manuscript.

\section{Acknowledgements}

This study owes a great deal to the management of waveform data by IRIS DMC, the NDIC's F-net in Japan, the J-Array Data Center, the Taiwan Data Center, and the ORPHEUS Data Center in Europe. Generic Mapping Tools (GMT) (Wessel and Smith 1995) were used for drawing all of the figures. Appreciation is extended to A. Souriau and G. Helffrich for providing individual S3KS-S2KS travel time measurement data. Thanks are also extended to George Helffrich who kindly checked our manuscript, and J. Ritsema who generously provided data from his tomography model. T. Tsuchiya is thanked for his enlightening discussions, and the comments and suggestions of Satoru Tanaka and the two anonymous reviewers were very helpful for improving the manuscript.

\section{Author details}

${ }^{1}$ Department of Earth and Planetary Science, Kyushu University, Hakozaki, Higashi-ku, 812-8581 Fukuoka, Fukuoka, Japan. ${ }^{2}$ National Research Institute for Earth Science and Disaster Prevention, 3-1 Tennodai, 305-0006 Tsukuba, Japan.

Received: 4 January 2015 Accepted: 14 May 2015

Published online: 24 June 2015

\section{References}

Alexandrakis C, Eaton DW (2010) Precise seismic-wave velocity atop Earth's core: No evidence for outer-core stratification. Phys Earth Planet Inter 180:59-65

Badro J, Cote AS, Brodholt JP (2014) A seismologically consistent compositional model of Earth's core. Proc Nat Acad Sci 111:7542-7545

Buffett BA, Seagle CT (2010) Stratification of the top of the core due to chemical interactions with the mantle. J Geophys Res 115. doi:10.1029/2009JB006751

Butler R, Anderson DL (1978) Equation of state fits to the lower mantle and outer core. Phys Earth Planet Inter 17:147-162

Choy G (1977) Theoretical seismograms of core phases calculated by a frequency-dependent full wave theory, and their interpretation. Geophys J Astr Soc 51:275-311

Dziewonski A, Anderson DL (1981) Preliminary reference Earth model. Phys Earth Planet Inter 25:297-356 
Eaton DW, Kendall J-M (2006) Improving seismic resolution of outermost core structure by multichannel analysis and deconvolution of broadband SmKS phases. Phys Earth Planet Inter 155:104-119

Garmany J, Orcutt JA, Parker RL (1979) Travel time inversion: a geometrical approach. J Geophys Res 84:3615-3622

Garnero EJ, Helmberger DV (1995) On seismic resolution of lateral heterogeneity in the Earth's outemost core. Phys Earth Planet Inter 88:117-130

Garnero EJ, Helmberger DV, Grand SP (1993) Constraining outermost core velocity with SmKS waves. Geophys. Res. Lett. 20:2463-2466

Helffrich G (2012) How light element addition can lower core liquid wave speeds. Geophys J Int 118:1065-1070

Helffrich, G (2014) Outer core compositional layering and constraints on core liquid transport properties. Earth Planet Sci Lett 391:256-262

Helffrich, G, Kaneshima S (2004) Seismological constraints on core composition from Fe-O-S liquid immiscibility. Science 306:2239-2242

Helffrich G, Kaneshima S (2010) Outer-core compositional stratification from observed core wave speed profiles. Nature 468:807-810

Helffrich G, Kaneshima S (2013) Cause and consequence of outer-core stratification. Phys Earth Planet Inter 223:2-7

Houser C, Masters G, Shearer P, Laske G (2008) Shear and compressional velocity models of the mantle from cluster analysis of long-period waveforms. Geophys J Int 174:195-212

Ichikawa H, Tsuchiya T, Tange Y (2014) The P-V-T equation of state and thermodynamic properties of liquid iron. J Geophys Res 119. doi:10.1002/2013JB010732

Kaneshima S, Helffrich G (2013) Vp structure of the outermost core derived from analyzing large-scale array data of SmKS waves. Geophys J Int 193:1537-1555

Kennett BLN, Engdahl ER (1991) Traveltimes for global earthquake location and phase identification. Geophys J Int 105:429-465

Kennett BLN, Engdahl ER, Buland R (1995) Constraints on seismic velocities in the Earth from traveltimes. Geophys J Int 126:108-124

Kind R (1979) Extensions of the reflectivity method for a buried source. J Geophys 45:373-380

Koci L, Belonoshko AB, Ahuja R (2007) Molecular dynamics calculation of liquid iron properties and adibatic temperature gradient in the Earth's outer core. Geophys J Int 168:890-894

Kustowski B, Ekstrom G, Dziewonski AM (2008) Anisotropic shear-wave velocity structure of the Earth's mantle. J Geophys Res 113:B06306

Lay T, Young C (1990) The stably-stratified outermost core revisited. Geophys. Res. Lett. 17:2001-2004

Lekic V, Cottaar S, Dziewonski AM, Romanowicz B (2012) Cluster analysis of global lower mantle tomography: a new structure and implications for chemical heterogeneity. Earth Planet Sci Lett 357-358:68-77

Mégnin C, Romanowicz B (2000) The shear velocity structure of the mantle from inversion of body, surface and higher modes waveforms. Geophys J Int 143:709-728

Morelli A, Dziewonski AM (1993) Body wave traveltimes and a spherically symmetric P- and S-wave velocity model. Geophys J Int 112:178-194

Obara K, Kasahara K, Hori S, Okada Y (2005) A densely distributed high sensitivity seismograph network in Japan: Hi-net by National Research Institute for Earth Science and Disaster Prevention. Rev Sci Intrum 76:021301

Pozzo M, Davies C, Gubbins D, Alfe D (2012) Thermal and electrical conductivity of iron at Earth's core conditions. Nature 485:355-358

Ritsema J, Deuss A, Heijst H, Woodhouse J (2011) S40RTS: a degree-40 shear-velocity model for the mantle from new Rayleigh wave dispersion, teleseismic traveltime and normal-mode splitting function measurements. Geophys J Int 184:1223-1236

Souriau A, Teste A, Chevrot S (2003) Is there any structure inside the liquid outer core? Goephys. Res. Lett. 30. doi:10.1029/2003GL017008

Takeuchi N (2007) Whole mantle SH velocity model constrained by waveform inversion based on 3D Born kernels. Geophys J Int 169:1153-1163

Tanaka S (2007) Possibility of a low P-wave velocity layer in the outermost core from global SmKS waveforms. Earth Planet Sci Lett 259:486-499

Vocadlo L, Dobson DP, Wood IG (2009) Ab initio calculations of the elasticity of hcp-Fe as a function of temperature at inner-core pressure. Earth Planet Sci Lett 288:534-538
Wang P, de Hoop MV, van der Hilst R (2008) Imaging the lowermost mantle (D") and the core-mantle boundary with SKKS coda waves. Geophys J Int 175:103-115

Wessel P, Smith WH (1995) New version of generic mapping tools. EOS Trans. AGU Electron. Suppl. Aug. 15

Yamanaka H, Ishida H (1995) Phase velocity inversion using genetic algorithms. J. Struct. Constr. Eng. AlJ 468:9-17

Zhang P, Cohen RE, Haule K (2015) Effects of electron correlations on transport properties of iron at Earth's core conditions. Nature 517:605-607

\section{Submit your manuscript to a SpringerOpen ${ }^{\mathcal{O}}$ journal and benefit from:}

- Convenient online submission

Rigorous peer review

- Immediate publication on acceptance

- Open access: articles freely available online

- High visibility within the field

- Retaining the copyright to your article

Submit your next manuscript at $>$ springeropen.com 